\title{
Design, synthesis and evaluation of anti-inflammatory, analgesic and antibacterial activity of 2, 4, 6-trisubstituted quinazoline derivatives
}

\author{
Nivrati JAIN, ${ }^{1}$ Harshita JAIN,${ }^{1}$ Ashish JAIN,${ }^{1}$ Veerasamy RAVICHANDRAN, ${ }^{2, *}$ and Prateek JAIN ${ }^{1, \dagger}$ \\ ${ }^{1}$ Adina Institute of Pharmaceutical Sciences, Sagar, (M.P) 470002, India \\ ${ }^{2}$ Faculty of Pharmacy, AIMST University, Semeling-08100, Kedah, Malaysia
}

\begin{abstract}
We described here the synthesis and biological evaluation of a series of 2, 4, 6-trisubstituted quinazoline derivatives as potential anti-inflammatory, analgesic and antibacterial agents. The synthesized compounds were characterized by FTIR, ${ }^{1} \mathrm{H}$ NMR and mass spectroscopy analysis. We found that the compounds $6 \mathrm{~b}, 6 \mathrm{e}, 6 \mathrm{~g}, 6 \mathrm{~h}$ and $6 \mathrm{j}$ showed better anti-inflammatory activity than indomethacin. Compounds $6 \mathrm{~b}, 6 \mathrm{e}, 6 \mathrm{~h}, 6 \mathrm{j}$ and 61 were found to exhibit appreciable analgesic activity, and 6b, 6g and 6k showed good antibacterial activity against Gram (+) bacteria: B. subtilis, S. aureus, S. epidermis, and Gram (-) bacteria: E. coli, P. aeruginosa and K. pneumoniae. Compound $6 \mathrm{~b}$ showed overall better anti-inflammatory, analgesic and antibacterial activity among the synthesized compounds. The results of the present study could be helpful for the designing of effective anti-inflammatory, analgesic and antibacterial agents.
\end{abstract}

Keywords: 2, 4, 6-trisubstituted quinazolines; anti-inflammatory; analgesic; antibacterial.

\section{Introduction}

Nonsteroidal anti-inflammatory drugs (NSAIDs) provide analgesic and antipyretic effects, which are widely used to treat acute and chronic inflammation [1]. NSAIDs show their effect by inhibition of COXs enzyme, which are used in prostaglandin biosynthesis from arachidonic acid. Both COX-1 and COX-2 enzymes are responsible for the production of prostaglandins that stimulate inflammation, pain, and fever. Nevertheless, the prostaglandins produced by COX-1 enzyme only activate platelets and protect the stomach and intestinal lining [24]. Gastrointestinal lesions, bleeding, nephrotoxicity and intestinal injury are the side effect of NSAIDs associated with long term clinical use. NSAIDs enteropathy is more frequent than NSAID gastropathy [5, 6]. Presences of the free carboxylic acid group in NSAIDs are responsible for their adverse reactions. The NSAIDs are widely used for the management of inflammation, pain, cardiovascular and genitourinary diseases $[7,8]$. Thus the synthesis of new compounds having better anti-inflammatory and analgesic activity with an improved safety profile is still necessary [9].

Derivatives of $4(3 \mathrm{H})$-quinazolinone [10], 4,6disubstituted quinazoline derivatives [9], 1-alkyl-4-aryl$2(1 \mathrm{H})$-quinazolines and quinazolinethiones [11], 2-(2,4disubstituted-thiazole-5-yl)-3-aryl-3H-quinazoline-4one derivatives [12], quinazoline and quinazolyl-4oxoquinazoline derivatives [13], substituted isoquinolines and quinazolines [14], 2,3-disubstituted quinazolin-4(3H)-ones [15] were reported to have significant anti-inflammatory, analgesic and antibacterial activities. "The halogen substituted heterocyclic moiety has attention due to the ability of halogen to act as polar hydrogen or hydroxyl mimic. Substitution of hydrogen by halogen has been a strategy in designing molecules for biological studies" [16]. Our hypothesis is based on the fact that the halogen on 6th position and substituted amine on 4th position of quinazoline nucleus may enhance their anti-inflammatory and analgesic activity with good antibacterial activity (Fig. 1).

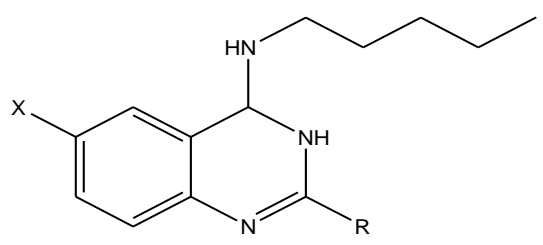

Figure 1. Structure of 2,4,6-trisubstituted quinazoline derivatives

\section{Experimental}

\subsection{Materials and methods}

The reaction progress was monitored by TLC on silica gel 60 F254 plates (Merck). The chromatograms were visualized under UV $254 \mathrm{~nm}$ and iodine. Melting points were determined by an open capillary method and were uncorrected and expressed in ${ }^{\circ} \mathrm{C}$. IR spectra of compounds were recorded on a Bruker ALPHA-T FTIR spectrometer using $\mathrm{KBr}$ disk. 1H NMR was recorded on Bruker-300 F MHz using TMS as an internal standard. All chemicals were purchased from $\mathrm{CDH}(\mathrm{P}) \mathrm{Ltd}$, India. Reagents and solvents used in the present study were mostly of LR (laboratory reagents) grade.

\subsection{Synthesis of 2, 4, 6, trisubstituted quinazoline derivatives \\ The synthesis of the 2, 4, 6-trisubstituted quinazoline derivatives 6 a to $6 \mathrm{n}$ was carried out by using the reported method with slight modification (Scheme 1) [16]. Briefly, the acid chloride (acetyl or benzoyl, $0.01 \mathrm{~mol}$ )}


was allowed to react with anthranilic acid or its 4 halogenated derivatives $(0.01 \mathrm{~mol})(1)$ in the presence of pyridine to yield benzoxazin-2-one derivatives (2). The corresponding phenylamidobenzamide derivatives (3) were obtained by stirring $2(0.01 \mathrm{~mol})$ with aqueous ammonia at room temperature for $4 \mathrm{~h}$. The excess ammonia was removed by evaporation and quenched with water. 2-Substituted quinazolin-4(3H)-ones (4) were obtained by refluxing phenylamidobenzamide derivatives $(0.01 \mathrm{~mol})$ in the presence of aqueous sodium hydroxide for $2 \mathrm{~h}$ and the reaction mixture was neutralized with acid. Then 2-substituted quinazolin$4(3 \mathrm{H})$-one $(0.01 \mathrm{~mol})$ was refluxed with phosphorous pentachloride $(0.05 \mathrm{~mol})$ and phosphorous oxychloride (0.05 mol) for $4 \mathrm{~h}$ at $115-118{ }^{\circ} \mathrm{C}$ to get 4-halo-2substituted quinazolines $(5)$.

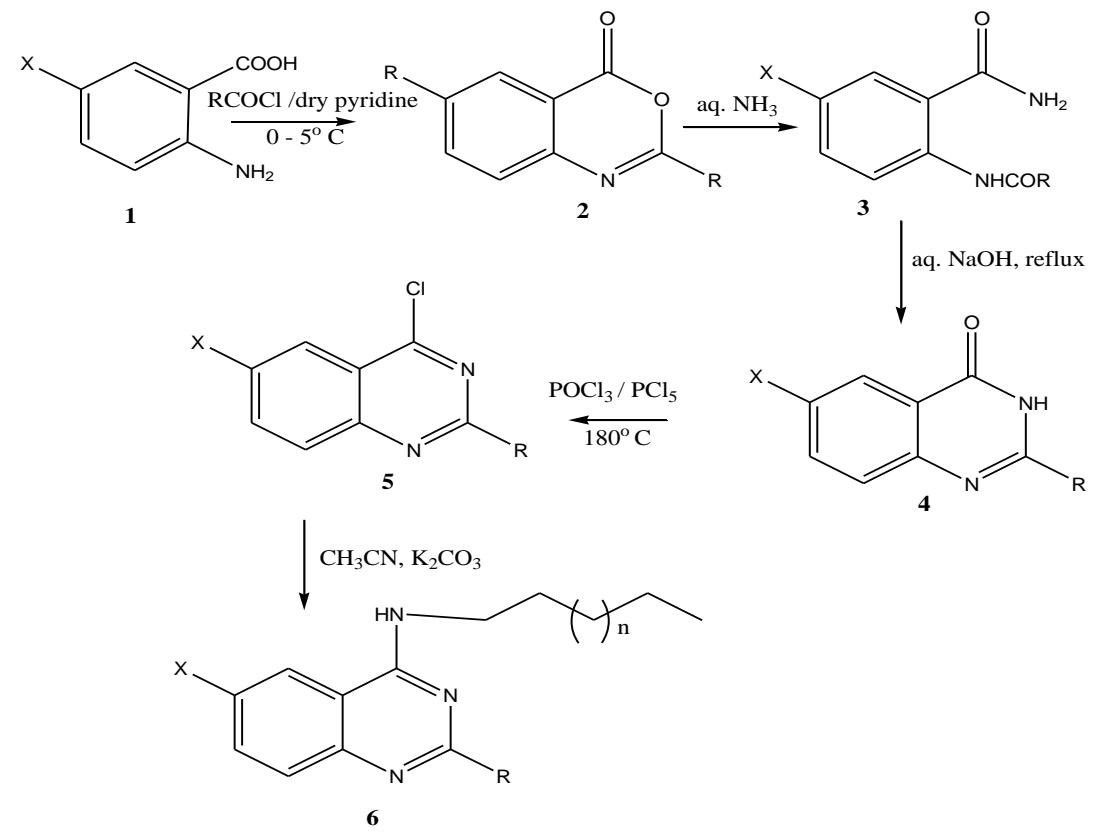

Scheme 1. Synthetic route of 2, 4, 6-trisubstituted quinazoline derivatives

The 4-halo-2-substituted quinazoline $(0.01 \mathrm{~mol})$ was dissolved in $(6 \mathrm{~mL})$ acetonitrile and added to equimolar quantity of 1-decylamine/ 1-hexylamine followed by potassium carbonate $(0.01 \mathrm{~mol})$ to get the final compounds 2,4,6-trisubstituted quinazoline. The reaction mixture was refluxed for $5 \mathrm{~h}$ at $80^{\circ} \mathrm{C}$ and cooled at room temperature. After removing the solvent under reduced pressure, the resultant residue was extracted with $15 \mathrm{~mL}$ of ethyl acetate and the extraction was repeated for 2-3 times. The combined organic extracts were washed with distilled water until the $\mathrm{pH}$ becomes neutral and then it was dried over anhydrous sodium sulfate. Finally, the organic extract was concentrated over rotavapor to get the final compounds of our interest (6a-n) and they were characterized by FTIR, ${ }^{1} \mathrm{H}$ NMR and mass spectroscopy analysis.

2.2.1. 6-(6-Bromo-2-methyl-4-quinazolinyl)- $N$ decylamine (6a). IR $\left(\mathrm{cm}^{-1}\right): 3200.02(\mathrm{Ar}-\mathrm{NH}), 2765.02$ (C-H Aromatic), $1650.68(\mathrm{C}=\mathrm{N}), 1438.25$ (aromatic C$\mathrm{N}), 550.52(\mathrm{C}-\mathrm{Br}) ;{ }^{1} \mathrm{H}$ NMR (DMSO- $\left.d_{6}\right) \delta \mathrm{ppm}: 0.98(\mathrm{~d}$, $\left.3 \mathrm{H}, \mathrm{CH}_{3}\right), 1.29$ (s, 14H, $\left.-\mathrm{CH}_{2}\right), 1.60$ (s, 2H, $\left.-13 \mathrm{NCR}\right)$, 2.45 (s, 3H, methyl), 7.90 (d, H, aromatic), 8.00 (d, 2H, methyl); Mass: $378.35\left(\mathrm{M}^{+}, 35 \%\right), 252.12,141,155$, $78.92,15$

2.2.2. 6-(6-Bromo-2-phenyl-4-quinazolinyl)- $N$ decylamine (6b). IR $\left(\mathrm{cm}^{-1}\right): 3226.02(\mathrm{Ar}-\mathrm{NH}), 3052.06$ (C-H aromatic), $1665.68(\mathrm{C}=\mathrm{N}), 1392.63$ (aromatic $\mathrm{C}-\mathrm{N})$, $550.52(\mathrm{C}-\mathrm{Br}) ;{ }^{1} \mathrm{H}$ NMR (DMSO- $\left.d_{6}\right) \delta \mathrm{ppm}: 0.98(\mathrm{~d}, 3 \mathrm{H}$, $\left.\mathrm{CH}_{3}\right), 1.33$ (s, 14H, $-\mathrm{CH}_{2}$ ), 1.53 (s, 2H, -1ß NCR), 4.00 (d, H, aromatic), 7.82-7.80 (d, 5H, benzene), $8.00(\mathrm{~d}, 3 \mathrm{H}$,
Ar- $\left.\mathrm{CH}_{2}\right)$; Mass: $440.42\left(\mathrm{M}^{+}, 65 \%\right), 314.13,141.77$, $78.91,77.7$

2.2.3. 6-(2-phenyl-4-quinazolinyl)- $N$-decylamine (6c). IR $\left(\mathrm{cm}^{-1}\right): 3224.24$ (Ar-NH), 2994.22 (C-H aromatic), $1642.84(\mathrm{C}=\mathrm{N}), 1015.05 ;{ }^{1} \mathrm{H}$ NMR $\left(\mathrm{DMSO}-d_{6}\right) \delta \mathrm{ppm}$ : 0.98 (d, 3H, $\left.\mathrm{CH}_{3}\right), 1.33$ (s, 14H, $\left.-\mathrm{CH}_{2}\right), 1.53$ (s, 2H, -1ß NCR), 2.36 (methyl), 4.04 (d, H, aromatic), 7.58 (1H, C$\mathrm{H}$, aromatic), $7.80(\mathrm{~d}, 5 \mathrm{H}$, benzene), $8.01(\mathrm{~d}, 3 \mathrm{H}, \mathrm{Ar}-$ $\mathrm{CH}_{2}$ ); Mass: $361.52\left(\mathrm{M}^{+}, 45 \%\right), 336,141.22,156,77.21$

2.2.4.

6-(6-Bromo-2-methyl-4-quinazolinyl)- $\mathrm{N}$ hexylamine (6d). IR $\left(\mathrm{cm}^{-1}\right)$ : $3200.02(\mathrm{Ar}-\mathrm{NH}), 1650.44$ $(\mathrm{C}=\mathrm{N}), \quad 1570.05 \quad(\mathrm{C}=\mathrm{C}), \quad 550.52 \quad(\mathrm{C}-\mathrm{Br}) ;{ }^{1} \mathrm{H} \quad \mathrm{NMR}$ (DMSO-d $) \delta$ ppm: $0.96\left(\mathrm{~d}, 3 \mathrm{H}, \mathrm{CH}_{3}\right), 1.33\left(\mathrm{~s}, 6 \mathrm{H},-\mathrm{CH}_{2}\right.$ ), 1.60 (s, 2H, -1ß NCR), 2.36 (3H,methyl), 3.90 (d, H, aromatic), 7.95 (d, 3H, Ar- $\left.\mathrm{CH}_{2}\right)$; Mass: $322.24\left(\mathrm{M}^{+}\right.$, $48 \%), 252.02,85,78.89,15.5$

2.2.5. 6-(6-Bromo-2-phenyl-4-quinazolinyl)- $\mathrm{N}$ hexylamine (6e). IR $\left(\mathrm{cm}^{-1}\right)$ : $3326.77(\mathrm{Ar}-\mathrm{NH}), 3070.73$ (C-H aromatic), $1680.68(\mathrm{C}=\mathrm{N}), 525.86(\mathrm{C}-\mathrm{Br}) ;{ }^{1} \mathrm{H}$ NMR (DMSO- $\left.d_{6}\right) \delta$ ppm: $0.98\left(\mathrm{~d}, 3 \mathrm{H}, \mathrm{CH}_{3}\right), 1.30\left(\mathrm{~s}, 6 \mathrm{H},-\mathrm{CH}_{2}\right)$, 1.52 (s, 2H, -1ß NCR), 4.00 (d, H aromatic), 7.48 (d, 5H, benzene), 8.00 (d, 3H, Ar- $\left.\mathrm{CH}_{2}\right)$; Mass: $384.31\left(\mathrm{M}^{+}\right.$, 67\%), 314.09, 85.5, 99.7, 78.98, 77.7

2.2.6. 6-(2-phenyl-4-quinazolinyl)- $N$-hexylamine (6f). IR $\left(\mathrm{cm}^{-1}\right)$ : 3344.24 (Ar-NH), 2932.22 (C-H aromatic), $1652.84(\mathrm{C}=\mathrm{N}), 1080.05 ;{ }^{1} \mathrm{H}$ NMR (DMSO- $\left.d_{6}\right) \delta \mathrm{ppm}$ : $0.98\left(\mathrm{~d}, 3 \mathrm{H}, \mathrm{B}-\mathrm{CH}_{3}\right), 1.30$ (s, 6H, $\left.-\mathrm{CH}_{2}\right), 1.52$ (s, 2H, -1ß NCR), 4.00 (d, H, aromatic), 7.32 (d, 5H benzene), 7.58 (1H,C-H, aromatic) $8.00\left(\mathrm{~d}, 3 \mathrm{H}, \mathrm{Ar}-\mathrm{CH}_{2}\right)$; Mass: 305.42 $\left(\mathrm{M}^{+}, 46 \%\right), 336.6,85.32,77.8$ 
2.2.7. 6-(6-Chloro-2-methyl-4-quinazolinyl)- $N$ decylamine (6g). IR ( $\left.\mathrm{cm}^{-1}\right): 3336.02$ (Ar-NH), 1660.44 $(\mathrm{C}=\mathrm{N}), 1556.55,671.64(\mathrm{C}-\mathrm{Cl}) ;{ }^{1} \mathrm{H}$ NMR (DMSO-d $\left.d_{6}\right) \delta$ ppm: 0.98 (d, 3H, $\left.3-\mathrm{CH}_{3}\right), 1.29$ (s, 14H, $\left.-\mathrm{CH}_{2}\right), 1.60$ (s, 2H, -1ß NCR), 2.45 (d, 2H, $\alpha$-methyl), 3.06 (3H, methyl), 3.90 (d, H, aromatic), 7.85 (d, 3H, benzene); Mass: 333.9 $\left(\mathrm{M}^{+}, 48 \%\right), 208.12,141.4,35.38,15.5$

2.2.8. 6-(6-Chloro-2-phenyl-4-quinazolinyl)- $N$ decylamine (6h). IR $\left(\mathrm{cm}^{-1}\right): 3252.02(\mathrm{Ar}-\mathrm{NH}), 3052.06$ (C-H Aromatic), 1363.63 (aromatic C-N), 525.52 (C-Cl); ${ }^{1} \mathrm{H}$ NMR (DMSO- $\left.d_{6}\right) \delta$ ppm: $0.98\left(\mathrm{~d}, 3 \mathrm{H}, \beta-\mathrm{CH}_{3}\right), 1.29$ (s, 14H, $-\mathrm{CH}_{2}$ ), 1.60 (s, 2H, -1ß NCR), 3.90 (d, H, aromatic), 7.32, 7.84 (d, 3H, benzene); Mass: 395.97 $\left(\mathrm{M}^{+}, 56 \%\right), 270.14,141.7,35.71,77.8$

2.2.9. 6-(6-Chloro-2-methyl-4-quinazolinyl)- $N$ hexylamine (6i). IR $\left(\mathrm{cm}^{-1}\right)$ : $3298.02(\mathrm{Ar}-\mathrm{NH}), 2700.02$ ($\mathrm{NH}) 1598.44(\mathrm{C}=\mathrm{N}), 698.52(\mathrm{C}-\mathrm{Cl})$; ${ }^{1} \mathrm{H}$ NMR (DMSO$\left.d_{6}\right) \delta$ ppm: 0.98 (d, 3H, ß-CH3), 1.29 (s, 6H, - $\left.\mathrm{CH}_{2}\right), 1.60$ (s, 2H, -1B NCR), 3.06 (3H, methyl), 3.90 (d, H, aromatic), $7.84\left(\mathrm{~d}, 3 \mathrm{H}\right.$, benzene); Mass: $277.79\left(\mathrm{M}^{+}\right.$, $57 \%), 208.66,85.8,35.56,15.2$

2.2.10. 6-(6- Chloro-2-phenyl-4-quinazolinyl)-Nhexylamine (6j). IR $\left(\mathrm{cm}^{-1}\right): 3240.24(\mathrm{Ar}-\mathrm{NH}), 2995.22$ (C-H Aromatic), $1642.84(\mathrm{C}=\mathrm{N}), 640.44(\mathrm{C}-\mathrm{Cl}) ;{ }^{1} \mathrm{H}$ NMR (DMSO- $\left.d_{6}\right) \delta$ ppm: $0.98\left(\mathrm{~d}, 3 \mathrm{H}, \beta-\mathrm{CH}_{3}\right), 1.29$ (s, $\left.6 \mathrm{H},-\mathrm{CH}_{2}\right), 1.60$ (s, 2H, -1ß NCR), 3.90 (d, H, aromatic), 7.32, $7.84\left(\mathrm{~d}, 3 \mathrm{H}\right.$, benzene); Mass: $339.86\left(\mathrm{M}^{+} 48 \%\right)$, $270.24,84.7,35.54,77.4$

2.2.11. 6-(6-Iodo-2-methyl-4-quinazolinyl)- $N$ decylamine (6k). IR $\left(\mathrm{cm}^{-1}\right): 3336.02(\mathrm{Ar}-\mathrm{NH}), 1660.44$ $(\mathrm{C}=\mathrm{N}), 1556.55(\mathrm{C}=\mathrm{C}), 541.42(\mathrm{C}-\mathrm{I})$; ${ }^{1} \mathrm{H}$ NMR (DMSO$\left.d_{6}\right) \delta$ ppm: $0.98\left(\mathrm{~d}, 3 \mathrm{H}, \mathrm{B}-\mathrm{CH}_{3}\right), 1.29\left(\mathrm{~s}, 14 \mathrm{H},-\mathrm{CH}_{2}\right), 1.60$ (s, 2H, -1ß NCR), 2.45 (d, 2H, $\alpha$ methyl), $3.06(3 \mathrm{H}$, methyl), 3.90 (d, H, aromatic), 8.21 (d, 3H, benzene); Mass: $425.35\left(\mathrm{M}^{+}, 39 \%\right), 300.22$, 141.6, 155.8, 127.02, 15

2.2.12.

6-(6-Iodo-2-phenyl-4-quinazolinyl)- $N$ decylamine (6l). IR $\left(\mathrm{cm}^{-1}\right)$ : $3252.72(\mathrm{Ar}-\mathrm{NH}), 2847.98$ ($\mathrm{NH}), 1651.72(\mathrm{C}=\mathrm{N}), 717.62(\mathrm{C}-\mathrm{I}) ;{ }^{1} \mathrm{H}$ NMR (DMSO$\left.d_{6}\right) \delta$ ppm: $0.98\left(\mathrm{~d}, 3 \mathrm{H}, \mathrm{B}-\mathrm{CH}_{3}\right), 1.29\left(\mathrm{~s}, 14 \mathrm{H},-\mathrm{CH}_{2}\right), 1.60$ (s, 2H, -1ß NCR), 3.90 (d, H, aromatic), 7.48, 8.21 (d, 3H, benzene); Mass: $487.42\left(\mathrm{M}^{+}, 58 \%\right), 362.21,141.5$, $126.78,78$

2.2.13. 6-(6-Iodo-2-methyl-4-quinazolinyl)- $N$ hexylamine $(6 \mathrm{~m})$. IR $\left(\mathrm{cm}^{-1}\right)$ : $3198.05(\mathrm{Ar}-\mathrm{NH}), 3015.01$ (C-H aromatic), $1680.68(\mathrm{C}=\mathrm{N}), 1576.95(\mathrm{C}=\mathrm{C}), 525.52$ (C-Br); ${ }^{1} \mathrm{H}$ NMR (DMSO-d $\left.d_{6}\right) \delta$ ppm: 0.98 (d, 3H, B $\left.\mathrm{CH}_{3}\right), 1.29$ (s, 6H, $\left.-\mathrm{CH}_{2}\right), 1.60$ (s, 2H, -1ß NCR), 2.35 (d, 2H, ó methyl), 3.06 (3H, methyl), 3.90 (d, H, aromatic), 8.11 (d, 3H, benzene); Mass: $369.24\left(\mathrm{M}^{+}, 68 \%\right), 300.12$, $85.7,99.9,127.9,15.4$

2.2.14. 6-(6-Iodo-2-phenyl-4-quinazolinyl)- $N$ hexylamine (6n). IR ( $\left.\mathrm{cm}^{-1}\right)$ : $3250.02(\mathrm{Ar}-\mathrm{NH}), 2744.02$ (C-H aromatic), $1660.44(\mathrm{C}=\mathrm{N}), 552.52(\mathrm{C}-\mathrm{I}) ;{ }^{1} \mathrm{H}$ NMR (DMSO-d $)_{6} \delta$ ppm: 0.98 (d, 3H, $\left.\beta-\mathrm{CH}_{3}\right), 1.29$ (s, 6H, $\mathrm{CH}_{2}$ ), 1.60 (s, 2H, -1ß NCR), 2.45 (d, 2H, ó methyl), 3.90 (d, H, aromatic), 7.33, $8.12(\mathrm{~d}, 3 \mathrm{H}$, benzene); Mass: $431.31\left(\mathrm{M}^{+}, 67 \%\right), 362.65,85.0,127.8,77.6$

\subsection{Microbiological assays}

All the synthesized compounds ( 6 a to $6 n$ ) were screened for anti-inflammatory, analgesic, antibacterial activity and for their acute toxicity.

2.3.1. Anti-inflammatory activity. The anti-inflammatory activity was evaluated by the carrageenan- induced paw edema techniques [17]. Using indomethacin as a reference drug and the test compounds were administered orally, at a dose of $100 \mathrm{mg} / \mathrm{kg}$ daily for 4 days, whereas the control group received the same volume of vehicle (Normal saline). The right hind paw volume was measured with plethysmometer before the carrageenan injection. After $2 \mathrm{~h}$ of drug administration, $0.2 \mathrm{~mL}$ of carrageenan $(10 \%, \mathrm{w} / \mathrm{v})$ was injected subcutaneously into the plantar surface of right hind paw and the paw volumes were measured after 2 and $3 \mathrm{~h}$. The anti-inflammatory effects (\% protection) of the test compounds were compared with that of control. The edema and inhibition rate were calculated by:

$$
\begin{gathered}
\text { Edema rate }(E)=\frac{V_{t}-V_{0}}{V_{0}} \\
\text { Inhibition rate }(I)=\frac{E_{c}-E_{t}}{E_{c}}
\end{gathered}
$$

where:

$V_{0}=$ the volume before carrageenan injection $(\mathrm{mL})$

$V_{\mathrm{t}}=$ the volume at $t \mathrm{~h}$ after carrageenan injection $(\mathrm{mL})$

$E_{\mathrm{c}}, E_{\mathrm{t}}=$ the edema rate of control group and treated group, respectively.

2.3.2. Analgesic activity. Test for analgesic activity was carried out by eddy`s hot plate method using hot-plate apparatus maintained at $53 \pm 0.5^{\circ} \mathrm{C}$ [18]. Swiss albino rats (25-35 g) of either sex were selected for the study. Diclofenac at a dose level of $100 \mathrm{mg} / \mathrm{kg}$, was administered orally as a reference drug. The test compounds $(10 \% \mathrm{v} / \mathrm{v}$ tween 80 suspensions) were administered orally at a dose level of $20 \mathrm{mg} / \mathrm{kg}$. The time interval between placing the animal in the hot plate and when it licked its hind paw or jumped was considered as the reaction time of the rat to the thermal stimulus. Basal reaction time was also measured prior to the administration of aqueous suspension of synthesized compounds and drug treatment $(0 \mathrm{~min})$. The reaction time was recorded at 30, 60 and $90 \mathrm{~min}$ after the treatment, and cut-off time was $10 \mathrm{~s}$. The analgesic effect was expressed as percentage of pretreatment level.

2.3.3. Acute toxicity. Approximately $50 \%$ lethal dose of the most promising analgesic and/or anti-inflammatory active agents determined by using Swiss albino rat (25$35 \mathrm{~g}$ ) of either sex were divided into 6 groups of 4 rat each. The compounds were dissolved in DMSO and injected at 100, 150, 200, 250 and $300 \mathrm{mg} / \mathrm{kg}$ doses. Then the mortality rates and toxic symptoms were recorded for $24 \mathrm{~h}$.

2.3.4. Antibacterial activity. The test compounds were screened for their antibacterial activity against $S$. aureus (ATCC29213), B. subtilis (ATCC6633), S. epidermis (ATCC35984), $K$. pneumoniae (ATCC13883), $P$. aeruginosa (ATCC27953), E. coli (ATCC 25922) by well diffusion method. The activities of test compounds were compared with that of reference drug streptomycin. 
The concentration of test compounds and standard drugs were $100 \mu \mathrm{g} / \mathrm{mL}$ and $50 \mu \mathrm{g} / \mathrm{mL}$, respectively. The diameter of the inhibition zone was measured at the end of $24 \mathrm{~h}$.

\section{Results and discussion}

The derivatives of 2,4,6-trisubstituted quinazoline were synthesized by simple method (Table 1) and the steps involved in the synthesis was illustrated in Scheme 1. The structures of the synthesized compounds (6a-n) were assigned by IR, ${ }^{1} \mathrm{H}$ NMR and mass spectroscopic data. ${ }^{1} \mathrm{H}$ NMR spectra of the 2,4,6-trisubstituted quinazoline derivatives (6a-n) showed a typical proton signal for the quinazoline ring at $7.80-8.25 \mathrm{ppm}$ range. In IR spectra of compounds (6a-n), the peak at 3200-3350 and 16401690 are attributed to Ar- $\mathrm{NH}$ and $\mathrm{C}=\mathrm{N}$, respectively. Mass spectra confirmed the molecular weight of the synthesized compounds and their fragmentation. Elemental analyses $(\mathrm{C}, \mathrm{H}, \mathrm{N})$ were in full agreement with the proposed structure of compounds and found to be within $\pm 0.4 \%$ of the theoretical values.

Table 1. Structural features of the synthesized 2,4,6trisubstituted quinazoline derivatives

\begin{tabular}{|cccccc|}
\hline $\begin{array}{c}\text { Comp. } \\
\text { No }\end{array}$ & $\mathbf{R}$ & $\mathbf{X}$ & $\boldsymbol{n}$ & $\begin{array}{c}\text { Yield } \\
(\mathbf{\%})\end{array}$ & $\begin{array}{c}\text { m.p. } \\
\left({ }^{\circ} \mathbf{C}\right)\end{array}$ \\
\hline $\mathbf{6 a}$ & $\mathrm{CH}_{3}$ & $\mathrm{Br}$ & 6 & 82 & 194 \\
$\mathbf{6 b}$ & $\mathrm{C}_{6} \mathrm{H}_{5}$ & $\mathrm{Br}$ & 6 & 80 & 168 \\
$\mathbf{6 c}$ & $\mathrm{C}_{6} \mathrm{H}_{5}$ & $\mathrm{H}$ & 6 & 78 & 186 \\
$\mathbf{6 d}$ & $\mathrm{CH}_{3}$ & $\mathrm{Br}$ & 2 & 81 & 115 \\
$\mathbf{6 e}$ & $\mathrm{C}_{6} \mathrm{H}_{5}$ & $\mathrm{Br}$ & 2 & 76 & 145 \\
$\mathbf{6 f}$ & $\mathrm{C}_{6} \mathrm{H}_{5}$ & $\mathrm{H}$ & 2 & 74 & 120 \\
$\mathbf{6 g}$ & $\mathrm{CH}_{3}$ & $\mathrm{Cl}$ & 6 & 72 & 125 \\
$\mathbf{6}$ & $\mathrm{C}_{6} \mathrm{H}_{5}$ & $\mathrm{Cl}$ & 6 & 71 & 117 \\
$\mathbf{6}$ & $\mathrm{CH}_{3}$ & $\mathrm{Cl}$ & 2 & 82 & 121 \\
$\mathbf{6 j}$ & $\mathrm{C}_{6} \mathrm{H}_{5}$ & $\mathrm{Cl}$ & 2 & 73 & 125 \\
$\mathbf{6 k}$ & $\mathrm{CH}_{3}$ & $\mathrm{I}$ & 6 & 71 & 115 \\
$\mathbf{6}$ & $\mathrm{C}_{6} \mathrm{H}_{5}$ & $\mathrm{I}$ & 6 & 72 & 120 \\
$\mathbf{6 m}$ & $\mathrm{CH}_{3}$ & $\mathrm{I}$ & 2 & 71 & 155 \\
$\mathbf{6 n}$ & $\mathrm{C}_{6} \mathrm{H}_{5}$ & $\mathrm{I}$ & 2 & 73 & 135 \\
\hline
\end{tabular}

The anti-inflammatory activity was assessed by carrageenan-induced paw edema techniques. Five of the synthesized compounds (6b, 6e, 6g, 6h and 6j) showed significant inhibition on carrageenan induced paw edema. It was observed that specifically compounds $6 \mathrm{e}$, $6 \mathrm{~h}$ and $6 \mathrm{j}$ were found to be significantly more potent than indomethacin (Table 2). Generally, compounds with phenyl substitution at position $R$ were more active than those with methyl group. Among the phenyl substituted (at position $R$ ) quinazolines, compound $6 \mathrm{j}$ showed remarkably significant inhibitory activity than the reference drug, which indicates that $R$ - phenyl, $\mathrm{X}-\mathrm{Cl}$ and $n=2$ are essential pharmacophores for antiinflammatory activity of the reported quinazoline derivatives. Among the methyl substituted (at position R) quinazolines, compounds $6 \mathrm{a}, 6 \mathrm{~g}$ and $6 \mathrm{k}$ showed better anti-inflammatory activity than others, it might be due to the presence of longer carbon chain in 4-amino substitution. Within the series of compounds, halogenated derivatives such as bromo and chloro derivatives exhibited better activity than the halogen free ones (6c and 6f).

Test for analgesic activity was carried out by Eddy`s hot plate method. It was noticed that the compounds $6 \mathrm{~b}$, $6 \mathrm{e}, 6 \mathrm{~h}, 6 \mathrm{j}$ and 61 showed significant analgesic effect than other molecules (Table 3). Generally, compounds with phenyl substitution at position $R$ were found to be remarkably active than those compounds with methyl substitution. Further substitution of decylamine on $4^{\text {th }}$ position showed a better analgesic effect than hexylamine.

Table 2. Anti-inflammatory activity of 2, 4, 6-trisubstituted quinazoline derivatives

\begin{tabular}{|ccc|}
\hline \multirow{2}{*}{ Compound } & \multicolumn{2}{c|}{ Percent protection $^{\mathrm{a}}$} \\
\cline { 2 - 3 } & $\mathbf{2 ~ h}$ & $\mathbf{3 ~ h}$ \\
\hline Control & $5 \% \pm 0.21$ & $13 \% \pm 0.49$ \\
Indomethacin & $57 \% \pm 1.21^{*}$ & $73 \% \pm 1.09^{* *}$ \\
$\mathbf{6 a}$ & $24 \% \pm 1.32^{* *}$ & $39 \% \pm 1.82^{*}$ \\
$\mathbf{6 b}$ & $52 \% \pm 1.42^{* *}$ & $69 \% \pm 1.21^{* *}$ \\
$\mathbf{6 c}$ & $14 \% \pm 1.11^{* *}$ & $23 \% \pm 1.45^{* *}$ \\
$\mathbf{6 d}$ & $21 \% \pm 1.13^{*}$ & $33 \% \pm 1.02^{*}$ \\
$\mathbf{6}$ & $59 \% \pm 1.03^{* *}$ & $76 \% \pm 1.08^{* *}$ \\
$\mathbf{6}$ & $22 \% \pm 1.42^{* *}$ & $41 \% \pm 1.72^{* *}$ \\
$\mathbf{6 g}$ & $47 \% \pm 1.05^{*}$ & $64 \% \pm 1.21^{* *}$ \\
$\mathbf{6 h}$ & $61 \% \pm 1.41^{*}$ & $79 \% \pm 1.54^{*}$ \\
$\mathbf{6} \mathbf{i}$ & $27 \% \pm 1.52^{*}$ & $38 \% \pm 1.43^{*}$ \\
$\mathbf{6 j}$ & $65 \% \pm 1.61^{* *}$ & $84 \% \pm 1.47 *$ \\
$\mathbf{6 k}$ & $25 \% \pm 1.13^{* *}$ & $48 \% \pm 1.23^{* *}$ \\
$\mathbf{6}$ & $26 \% \pm 1.24 *$ & $47 \% \pm 1.43^{* *}$ \\
$\mathbf{6 m}$ & $34 \% \pm 1.64 *$ & $45 \% \pm 1.81^{* *}$ \\
$\mathbf{6 n}$ & $25 \% \pm 1.07 * *$ & $36 \% \pm 1.04 *$ \\
\hline
\end{tabular}

Each value represents the mean $\pm \operatorname{SD}(n=6)$.

$*$ Significantly different from control at $p<0.05$; **Significantly different from control at $p<0.01 ;{ }^{\text {a }}$ - Percent inhibition of carrageenaninduced edema measured after $2 \mathrm{~h}$ and $3 \mathrm{~h}$.

In terms of duration of analgesia it was observed that all test compounds exhibited analgesia for more than $2 \mathrm{~h}$ whereas compound $6 \mathrm{~b}$ and $6 \mathrm{e}$ showed potent analgesia for more than $3 \mathrm{~h}$. It could be postulated that peripheral or cerebral mechanisms might be involved in the inhibitory effects of these compounds. Among the phenyl substituted (at position $R$ ) quinazolines, compound $6 \mathrm{~b}$ showed excellent inhibitory activity than the reference drug diclofenac, and the other compounds $6 \mathrm{e}$ and $6 \mathrm{j}$ were almost active as diclofenac, which indicates that $R-$ phenyl, $\mathrm{X}-\mathrm{Cl}$ and $n=2$ are essential pharmacophores for analgesic activity of quinazoline derivatives. It was noted that the compounds with bromo substitution were more active than other derivatives in the series. The ability of compounds to inhibit both pain and inflammation greatly reflects the involvement of prostaglandin inhibition in the observed actions.

Table 3. Analgesic activity of synthesized 2,4,6 - trisubstituted quinazoline derivatives

\begin{tabular}{|c|c|c|c|c|}
\hline \multirow[t]{2}{*}{ Compound } & \multirow{2}{*}{$\begin{array}{l}\text { Basal reaction time }(\mathrm{sec}) \\
\text { before treatment } \\
(\text { mean } \pm \text { SD })\end{array}$} & \multicolumn{3}{|c|}{$\begin{array}{l}\text { Basal reaction time }(\text { min) after treatment } \\
\qquad(\text { mean } \pm \text { SD) }\end{array}$} \\
\hline & & $30 \mathrm{~min}$ & $60 \mathrm{~min}$ & $90 \mathrm{~min}$ \\
\hline Control & $3.65 \pm 0.676$ & $3.42 \pm 0.526$ & $3.38 \pm 0.5266$ & $3.21 \pm 0.4141$ \\
\hline Diclofenac & $3.85 \pm 0.562$ & $10.45 \pm 0.642 * *$ & $11.32 \pm 0.691 * *$ & $13.45 \pm 0.731 * *$ \\
\hline $6 a$ & $3.12 \pm 0.546$ & $7.24 \pm 0.212 *$ & $8.34 \pm 0.341 * *$ & $8.98 \pm 0.346 * *$ \\
\hline
\end{tabular}




\begin{tabular}{|c|c|c|c|c|}
\hline \multirow[t]{2}{*}{ Compound } & \multirow{2}{*}{$\begin{array}{l}\text { Basal reaction time }(\mathrm{sec}) \\
\text { before treatment } \\
(\text { mean } \pm \text { SD })\end{array}$} & \multicolumn{3}{|c|}{$\begin{array}{l}\text { Basal reaction time }(\min ) \text { after treatment } \\
(\text { mean } \pm \text { SD) }\end{array}$} \\
\hline & & $30 \mathrm{~min}$ & $60 \mathrm{~min}$ & $90 \mathrm{~min}$ \\
\hline $6 \mathbf{6 b}$ & $3.01 \pm 0.512$ & $11.42 \pm 0.762 * *$ & $12.62 \pm 0.812 * *$ & $12.98 \pm 0.892 * *$ \\
\hline 6c & $4.65 \pm 0.6766$ & $5.32 \pm 0.762$ & $6.54 \pm 0.662$ & $7.65 \pm 0.762 *$ \\
\hline 6d & $3.65 \pm 0.541$ & $4.64 \pm 0.762$ & $5.12 \pm 0.462$ & $5.98 \pm 0.614$ \\
\hline $6 e$ & $4.15 \pm 0.561$ & $11.52 \pm 0.785^{* *}$ & $11.98 \pm 0.792 * *$ & $12.65 \pm 0.811 * *$ \\
\hline 6f & $3.16 \pm 0.561$ & $4.98 \pm 0.762$ & $5.65 \pm 0.762$ & $5.98 \pm 0.612$ \\
\hline $6 \mathrm{~g}$ & $3.05 \pm 0.261$ & $8.38 \pm 0.412 * *$ & $8.82 \pm 0.465 * *$ & $7.54 \pm 0.321 *$ \\
\hline $6 \mathrm{~h}$ & $3.60 \pm 0.612$ & $9.34 \pm 0.498 * *$ & $10.67 \pm 0.681 * *$ & $11.23 \pm 0.691 * *$ \\
\hline $6 \mathbf{i}$ & $4.02 \pm 0.676$ & $7.35 \pm 0.225 *$ & $8.45 \pm 0.321 * *$ & $7.87 \pm 0.310 *$ \\
\hline $6 \mathbf{j}$ & $4.23 \pm 0.716$ & $10.32 \pm 0.592 * *$ & $11.56 \pm 0.587 * *$ & $12.34 \pm 0.612 * *$ \\
\hline $6 \mathbf{k}$ & $3.52 \pm 0.576$ & $5.86 \pm 0.762$ & $6.89 \pm 0.812$ & $7.34 \pm 0.821 *$ \\
\hline 61 & $3.44 \pm 0.623$ & $9.33 \pm 0.487 * *$ & $9.98 \pm 0.542 * *$ & $10.34 \pm 0.597 * *$ \\
\hline $6 m$ & $3.04 \pm 0.446$ & $7.34 \pm 0.231 *$ & $8.98 \pm 0.342 * *$ & $8.65 \pm 0.321 * *$ \\
\hline $6 n$ & $3.12 \pm 0.611$ & $6.78 \pm 0.312$ & $7.67 \pm 0.432 *$ & $8.12 \pm 0.498 * *$ \\
\hline
\end{tabular}

Each value represents the mean $\pm \operatorname{SD}(n=6)$.

$*$ Indicates the significant difference compared with control, $p<0.05 ; * *$ Indicates the highly significant difference compared with control, $p<0.001$.

The compounds which exhibited significance antiinflammatory, analgesic and antibacterial $(6 \mathrm{~b}, 6 \mathrm{e}, 6 \mathrm{~h}, 6 \mathrm{j}$ and 61) were subjected to toxicological studies using $\mathrm{LD}_{50}$ standard method in rat at a concentration of 100 , 150, 200, 250 and $300 \mathrm{mg} / \mathrm{kg}$ (body weight). No mortality or toxic symptoms were observed in test animals within $24 \mathrm{~h}$ post-administration. It was noticed that the compounds $6 \mathrm{e}, 6 \mathrm{j}$ and 61 induced urination whereas compounds $6 \mathrm{~b}$ and $6 \mathrm{~h}$ induced sedation, calmness, muscle relaxation and decreased respiration. Even though, no mortality rates or toxic symptoms were observed in test animals after $24 \mathrm{~h}$.

The test compounds were screened for their in vitro antibacterial activity using well diffusion method.
Compounds $(6 \mathrm{~b}, 6 \mathrm{~g}, 6 \mathrm{k})$ showed good activity against Gram-positive bacteria, and compounds $(6 \mathrm{~g}, 6 \mathrm{~h}, 6 \mathrm{k})$ exhibited activity against Gram-negative bacterial strains (Table 4). It should also be noted that halogen substituted compounds at $6^{\text {th }}$ position give better activity than halogen free compounds. Among all the active compounds, compound $6 \mathrm{~g}$ showed better inhibitory activity against both Gram-positive and negative strains and its inhibitory activity was significantly more potent than streptomycin. Results clearly suggest that $R-$ methyl, $\mathrm{X}-\mathrm{Cl}$ and $n=6$ are essential pharmacophores for antibacterial activity of quinazoline derivatives.

Table 4. The antibacterial activity of 2,4,6-trisubstituted quinazoline derivatives compounds

\begin{tabular}{|c|c|c|c|c|c|c|}
\hline \multirow{2}{*}{$\begin{array}{c}\text { Compd. } \\
(100 \mu \mathrm{g} / \mathrm{mL})\end{array}$} & \multicolumn{3}{|c|}{$\begin{array}{c}\text { Gram (+) strains } \\
\text { Zone of inhibition }(\mathbf{m m})\end{array}$} & \multicolumn{3}{|c|}{$\begin{array}{c}\text { Gram (-) strains } \\
\text { Zone of inhibition }(\mathbf{m m})\end{array}$} \\
\hline & B. subtilis & S. aureus & S. epidermis & E. coli & P. aeruginosa & K. pneumoniae \\
\hline $6 a$ & 15 & 11 & 16 & 08 & 12 & 10 \\
\hline $6 \mathbf{b}$ & 23 & 20 & 24 & 27 & 15 & 16 \\
\hline $6 c$ & 18 & 12 & 08 & 09 & 15 & 12 \\
\hline 6d & 12 & 08 & 14 & 11 & 10 & 09 \\
\hline $6 e$ & 09 & 09 & 15 & 12 & 13 & 14 \\
\hline 6f & 08 & 10 & 09 & 08 & 09 & 11 \\
\hline $6 \mathrm{~g}$ & 25 & 23 & 22 & 30 & 26 & 24 \\
\hline $6 \mathrm{~h}$ & 10 & 13 & 12 & 23 & 21 & 17 \\
\hline $6 \mathbf{i}$ & 12 & 14 & 11 & 17 & 16 & 15 \\
\hline $6 \mathbf{j}$ & 11 & 10 & 14 & 13 & 21 & 18 \\
\hline $6 \mathbf{k}$ & 25 & 19 & 22 & 28 & 21 & 24 \\
\hline 61 & 12 & 15 & 20 & 17 & 18 & 08 \\
\hline $6 \mathrm{~m}$ & 21 & 18 & 16 & 20 & 12 & 20 \\
\hline $6 n$ & 19 & 09 & 21 & 18 & 12 & 16 \\
\hline $\begin{array}{l}\text { Streptomycin } \\
(50 \mu \mathrm{g} / \mathrm{mL})\end{array}$ & 24 & 21 & 23 & 29 & 24 & 23 \\
\hline
\end{tabular}

\section{Conclusion}

In the present study, a range of 2,4,6-trisubstituted quinazoline derivatives were successfully synthesized and screened for their anti-inflammatory, analgesic, antibacterial activity and acute toxicity. The tested compounds showed comparable activities with respective standard drug. The results of the present study supports our hypothesis that halogen on $6^{\text {th }}$ position and substituted amine on the $4^{\text {th }}$ position of quinazoline nucleus may enhance their anti-inflammatory and analgesic activity with good antibacterial activity. Based on the current study, in future the compound $6 \mathrm{~b}$ will be optimized as potential lead for anti-inflammatory and analgesic activity, and compound $6 \mathrm{~g}$ will be optimized as potential lead for antibacterial activity.

\section{Conflict of interest}

The authors confirm that this article content has no conflicts of interest. 


\section{References}

[1]. B.L. SNG, S.A. Schug, The role of opioids in managing chronic non-cancer pain, Ann. Acad. Med. 38 (2009) 960 - 966.

[2]. A.M. Jane, D.W. Timothy, Cyclo-oxygenase-2: pharmacology, physiology, biochemistry and relevance to NSAID therapy, Br. J. Pharmacol. 128 (1999) 1121-1132.

[3]. J.R. Vane, Y.S. Bakhle, R.M. Botting, Cyclooxygenases 1 and 2, Ann. Rev. Pharmacol. Toxicol. 38 (1998) 97-120.

[4]. D.L. Simmons, R.M. Botting, T. Hla, Cyclooxygenase isozymes: The biology of prostaglandin synthesis and inhibition, Pharmacol. Rev. 56 (2004) 387 - 437.

[5]. D. Adebayo, I. Bjarnason, Is non-steroidal antiinflammaory drug (NSAID) enteropathy clinically more important than NSAID gastropathy?, Postgrad. Med. J. 82 (2006) 186 - 191.

[6]. S.C. Park, H.J. Chun, C.D. Kang, D. Sul, Prevention and management of non-steroidal antiinflammatory drugs-induced small intestinal injury, World J. Gastroenterol. 17 (2011) 4647-4653.

[7]. A. Mishra, R. Veerasamy, P.K. Jain, V.K. Dixit, R.K. Agrawal, Synthesis, characterization and pharmacological evaluation of amide prodrugs of Flurbiprofen, J. Braz. Chem. Soc. 19 (2008) 89 100.

[8]. A. Mishra, R. Veerasamy, P.K. Jain, V.K. Dixit, R.K. Agrawal, Synthesis, characterization and pharmacological evaluation of amide prodrugs of ketorolac, Eur. J. Med. Chem. 43 (2008) 2464 2472.

[9]. P.M. Chandrika, T. Yakaiah, A.R.R. Rao, B. Narsaiah, N.C. Reddy, V. Sridhar, J.V.E. Rao, Synthesis of novel 4,6-disubstituted quinazoline derivatives, their anti-inflammatory and anti-cancer activity (cytotoxic) against U937 leukemia cell lines, Eur. J. Med. Chem. 43 (2008) 846 - 852.

[10]. A.A. Farag, E.M. Khalifa, N.A. Sadik, S.Y. Abbas, A.G. Alsehemi, A.G. Ammar, Synthesis, characterization, and evaluation of some novel $4(3 \mathrm{H})$-quinazolinone derivatives as antiinflammatory and analgesic agents, Med. Chem. Res. 22 (2013) 440 - 452.
[11]. R.V. Coombs, R.P. Danna, M. Denzer, G.E. Hardtmann, B. Huegi, G. Koletar, J. Koletar, H. Ott, E. Jukniewicz, Synthesis and antiinflammatory activity of 1-alkyl-4-aryl-2(1H)-quinazolines and quinazolinethiones, J. Med.Chem. 16 (1973) 1237 1245.

[12]. R.S. Giri, H.M. Thaker, T. Giordano, J. Williams, D. Rogers, V. Sudersanam, K.K. Vasu, Design, synthesis and characterization of novel 2-(2, 4disubstituted-thiazole-5-yl)-3-aryl-3H-quinazoline4-one derivatives as inhibitors of NF- $\mathrm{\kappa B}$ and AP-1 mediated transcription activation and as potential anti-inflammatory agents, Eur. J. Med. Chem. 44 (2009) 2184 - 2189.

[13]. M.M. Gineinah, M.A. El-Sherbeny, M.N. Nasr, A.R. Maarouf, Synthesis of antiinflammatory screening of some quinazoline derivatives, Arch. Pharm. Pharm. Med. Chem. 11 (2002) 556 - 562.

[14]. Q. Chao, L. Deng, H. Shih, L.M. Leoni, D. Genini, D.A. Carson, H.B. Cottam, Substituted isoquinolines and quinazolines as potential antiinflammatory agents. Synthesis and biological evaluation of inhibitors of tumor necrosis factor $\alpha$, J. Med. Chem. 42 (1999) 3860 - 3873.

[15]. V. Alagarsamy, V. Muthukumar, M. Pavalarani, P. Vasanthanathan, R. Revathi, Synthesis, analgesic and anti-inflammatory activities of some novel 2,3disubstituted quinazolin-4(3h)-ones, Biol. Pharm. Bull. 2003, 26, 557 - 559.

[16]. A.M. Alafeefy, A.A. Kadi, O.A. Al-Deeb, K.E. ElTahir, N.A. Al-Jaber, Synthesis, analgesic and antiinflammatory evaluation of some novel quinazoline derivatives, Eur. J. Med. Chem. 11 (2010) 4947 4952.

[17]. S.M. Perez, M. Perez, A. Susunaga, M.A. Zavala, Anti-inflammatory activity of Lippia dulcis, J. Ethanopharmacol. 102 (2005) 1-4.

[18]. J. Silva, W. Abebeb, S.M. Sousa, V.G. Duarte, M.I.L. Machadoc, F.J.A. Matos, Analgesic and anti-inflammatory effects of essential oils of eucalyptus, J. Ethanopharmacol. 89 (2003) 277283.

Received: 09.08.2018

Received in revised form: 27.09.2018

Accepted: 01.10.2018 